\title{
Numerical Models of Galactic Dynamos
}

\author{
Detlef Elstner \\ Astrophysikalisches Institut Potsdam, An der Sternwarte 16, 14482 \\ Potsdam, Germany
}

\begin{abstract}
The state of the art for dynamo models in spiral galaxies is reviewed. The comparison of numerical models with special properties of observed magnetic fields yields constraints for the turbulent diffusivity and the $\alpha$-effect. The derivation of the turbulence parameters from the vertical structure of the interstellar medium gives quite reasonable values for modelling the regular magnetic fields in galaxies with an $\alpha^{2} \Omega$-dynamo. Considering the differences of the turbulence between spiral arms and interarm regions, the observed interarm magnetic fields are recovered in the numerical models due to the special properties of the $\alpha^{2} \Omega$-dynamo.
\end{abstract}

The first kinematic models for galactic dynamos in an ellipsoidal geometry were presented by Stix (1978) and White (1978). Deinzer et al. (1990) investigated numerical models in a torus geometry. Elstner et al. (1990) introduced the concept of non-sharp boundaries, describing galaxies by diffusivityand $\alpha$-profiles. With a similar approach Donner \& Brandenburg (1990) presented dynamo excited magnetic field configurations and calculated the polarized emission. In a series of kinematic models for different galaxies Elstner et al. (1992) found only axisymmetric solutions. The observed pitch angles could only be reproduced with models where $\alpha$ is of the order of $10 \mathrm{~km} \mathrm{~s}^{-1}$ and the diffusivity about $1 \mathrm{kpc}^{2} \mathrm{Gyr}^{-1}$. Under these conditions the dynamo operates in an $\alpha^{2} \Omega$-regime, which results in a stationary quadrupole. In order to explain observations of vertical fields above the disk of NGC4631, models with more elaborated turbulent coefficients and additional mean velocity terms like wind flows were studied (Brandenburg et al. 1993; Elstner et al. 1995). The explanation of the bisymmetric field in M81 was still an unresolved problem. With the first 3D models Moss et al. (1993) gave a possible explanation with the combined effect of a bisymmetric seed field, nonaxisymmetric turbulence and mean flow caused by an encounter with a companion. Including spiral flows in their models Panesar, Moore \& Nelson (1993) found magnetic field concentration in the spiral arms. Energetic arguments prefer supernova explosions as driver for the turbulence. Ziegler (1996) first calculated the turbulent coefficients and the back reaction of the mean field in such a scenario. The results were similar to that derived with SOCA. Later models with quenching of the diffusivity (Elstner et al. 1996) had problems in explaining the saturation of magnetic fields at equipartition. The nonlinear problem is still under discussion and possibly magnetic instabilities should be considered as another source for turbulence. Nevertheless already the kinematic solutions agree quite well with observations. All nonlinear effects considered in the models do not qualitatively change the magnetic field structure. Magnetic arms between optical arms as observed in 
NGC6946 could be understood mainly due to different diffusivities in arms and interarm regions (Rohde et al. 1999). Recent models with more realistic spiral velocities obtained from hydro-simulations show a clear connection of magnetic field structure with the spiral structure of the gas (Elstner et al. 2000). With a similar technique magnetic fields in barred galaxies also have been investigated (Otmianowska-Mazur et al. 1997; Moss et al. 1999). Because no variation of the turbulence velocity $u^{\prime}$ is observed in NGC6946, different turbulence properties in arms and interarms can only be due to the correlation length $l$ and correlation time $\tau$. Assuming larger structures in the arms (super-bubbles) combined with a larger $\tau$, a larger dynamo number $D=C_{\alpha} C_{\Omega}-C_{\alpha}^{2}=\Omega^{2} H^{2} / u^{\prime 2}-\tau^{2} \Omega^{2}$ results in the interarms for the $\alpha^{2} \Omega$-dynamo. Taking the observed rotation $\Omega$ and $u^{\prime}$ at the midplane and calculating the vertical structure by assuming a vertical density stratification as in our galaxy, $\tau$ remains as the only free parameter. With an azimuthal variation of $\tau$ from 10 to 20 million years a good agreement with observations is achieved (Rohde et al. 1999). Spiral flows from hydro-simulations give enhanced magnetic arms in the optical arms. Both effects together should explain the zoo of magnetic fields in real galaxies.

\section{References}

Brandenburg, A., Donner, K. J., Moss, D., Shukurov, A., Sokoloff, D. D. \& Tuominen, I. 1993, A\&A, 273, 36

Deinzer, W., Grosser, H. \& Schmitt, D. 1990, A\&A, 273, 405

Donner, K. J. \& Brandenburg, A. 1990, A\&A, 240, 289

Elstner, D., Meinel, R. \& Rüdiger, G. 1990, GApFD, 50, 85

Elstner, D., Meinel, R. \& Beck, R. 1992, A\&AS, 94, 587

Elstner, D., Golla, G., Rüdiger, G. \& Wielebinski, R. 1995, A\&A, 297, 77

Elstner, D., Rüdiger, G. \& Schultz, M. 1996, A\&A, 306, 740

Elstner, D., Otmianowska-Mazur, K., von Linden, S. \& Urbanik, M. 2000, A\&A, 357,129

Moss, D., Brandenburg, A., Donner, K. J. \& Thomasson, M. 1993, ApJ, 409, 179

Moss, D., Rautiainen, P. \& Salo, H. 1999, MNRAS, 303, 125

Otmianowska-Mazur, K., von Linden, S., Lesch, H. \& Skupniewicz, G. 1997, $A \& A, 323,560$

Panesar, J.S., Moore, N. \& Nelson, A.H. 1993, in IAU Symp 157, The cosmic dynamo, ed. F. Krause, K. H. Rädler \& G. Rüdiger (Dordrecht: Kluwer), 345

Rohde, R., Beck, R. \& Elstner, D. 1999, A\&A, 350, 423

Stix, M. 1978, A\&A, 68, 459

White, M.P. 1978, Astronomische Nachrichten, 299, 209

Ziegler, U. 1996, A\&A, 313, 448 